\title{
Fatty Acid Profiles in Relation to Triglyceride Level in the Liver of Dairy Cows
}

\author{
Hiroshi SATO $^{1)}$, Tharwat MOHAMED ${ }^{1)}$, Akiko GOTO ${ }^{1)}$, Shin OIKAWA ${ }^{1)}$ and Takashi KUROSAWA ${ }^{1)}$ \\ ${ }^{1)}$ Department of Veterinary Internal Medicine, School of Veterinary Medicine, Rakuno Gakuen University, Ebetsu, Hokkaido 069-8501, \\ Japan
}

(Received 5 June 2003/Accepted 20 August 2003)

ABSTRACT. To elucidate possible relationships between triglyceride (TG) levels and fatty acid composition in bovine liver, hepatic TG and seven individual fatty acids were measured in 23 Holstein dairy cows, of them 6 are healthy. Liver TG level was greater than $3 \%$ in 12 cows which were ruled fatty liver. Palmitic and oleic acid proportions were significantly higher in fatty liver cows than in the healthy cows, while stearic acid was lower in fatty liver cows. With increased liver TG, stearic acid proportions decreased dramaticall y. Results indicate that hepatic lipidosis markedly alters the proportions of the various fatty acids in the liver of dairy cows.

KEY WORDS: dairy cow, fatty acid, fatty liver.

J. Vet. Med. Sci. 66(1): 85-87, 2004

Nonesterified fatty acids (NEFA) are mobilized from the adipose tissue to yield energy during periods of negative energy balance. Given that the liver is an important organ for taking up circulating NEFA in ruminants [1,3], the liver accumulates high amounts of triglyceride (TG) in cows having conditions associated with nutritional deficiency [1-3, $7,8]$. In cattle, much information is available on the fatty acid proportions in soft tissue such as muscle and adipose tissue [12]. Little is available, however, on the fatty acid profiles and metabolic behavior of the individual fatty acids in the liver, particularly in dairy cows with fatty liver [7,8]. The purpose of the present study was to evaluate the proportions of the various fatty acids in the liver, especially in cows with increased hepatic TG.

Twenty-three Holstein cows were used, including 6 healthy and 17 with clinical disorders such as respiratory dysfunctions (6 cases), postpartum anorexia (2), locomotive dysfunctions (4), digestive or peritoneal disorders (4) and a ligament rupture of the udder (1). All the cows were euthanized for pathological anatomy. Care and handling of the animals were in accordance with the Laboratory Animal Control Guidelines of Rakuno Gakuen University, which are in conformity with the Guidelines for the Care and Use of Laboratory Animals of the National Institutes of Health, U.S.A.

Liver tissues were sampled from the right, left and qua- dratic lobes at necropsy and frozen at $-30^{\circ} \mathrm{C}$ until analysis. After the liver lipids were extracted by isopropanol -heptane and the polar lipids were removed by alumina absorption, the TG level in each lobe was determined by acetylacetone color development; the mean TG level was calculated per each cow. For determining the individual fatty acid in the liver, whole lipid was extracted by chlorofolm -methanol. The extracted lipids were methylated by strong agitation with sodium methylate in methanol. The methyl esters were analyzed for individual fatty acids by gas chromatography (Shimadzu GC-9A; Kyoto) with a flame ionization detector. In the present study, a TG level of greater than $3 \%$ was considered to indicate fatty liver. According to this criterion, fatty liver was confirmed in 12 cows of 17 clinical cases, including one with focal fatty liver in the left lobe. Liver fatty acid proportions and TG levels were compared between fatty liver cows and healthy cows by $t$-test (Table 1). The other 5 cows of 17 clinical cases were not suffered from fatty liver. To determine the relationships between the TG levels and fatty acid proportions of the liver, scatter graph was drawn based on all the 23 cows.

Liver TG ranged from 0.13 to $0.32 \%$ in the healthy cows, and from 4.0 to $36.4 \%$ in the fatty liver cows $(P<0.001)$. Proportion of stearic acid was significantly lower $(P<0.001)$, whereas palmitic and oleic acids were significantly higher $(P<0.001,0.01)$ in fatty liver cows than in

Table 1. Fatty acid profiles compared in the liver of fatty liver cows and healthy cows

\begin{tabular}{|c|c|c|c|c|c|c|c|c|c|}
\hline & & \multirow{2}{*}{$\begin{array}{l}\text { Liver } \mathrm{TG}^{\mathrm{a})} \\
(\% \bullet \text { fresh })\end{array}$} & \multicolumn{7}{|c|}{ (\% in total 7 fatty acids) } \\
\hline & & & C 14:0 & C 16:0 & C $16: 1$ & C 18:0 & C $18: 1$ & C $18: 2$ & C $18: 3$ \\
\hline Fatty liver cows ${ }^{b)}$ & Mean & $12.9 * * *$ & 2.9 & $38.9 * * *$ & 5.3 & $7.7 * * *$ & $37.9 * *$ & 6.9 & 0.5 \\
\hline$(n=12)$ & SD & 10.6 & 0.7 & 5.4 & 2.4 & 1.6 & 4.4 & 2.6 & 0.4 \\
\hline Healthy cows & Mean & 0.2 & 2.1 & 25.4 & 5.3 & 28.5 & 29.2 & 9.4 & 0.1 \\
\hline$(n=6)$ & SD & 0.1 & 0.5 & 2.3 & 1.6 & 3.8 & 3.5 & 3.3 & 0.1 \\
\hline
\end{tabular}

C14:0 Myristic acid, C16:0 Palmitic acid, C16:1 Palmitoleic acid, C18:0 Stearic acid, C18:1 Oleic acid, C18:2 Linoleic acid, C18:3 Linolenic acid.

a) Triglyceride b) Liver TG $\geq 3 \%$ (fresh). Significant difference compared to healthy cows at $P<0.01(* *)$ and $P<0.001(* * *)$. 

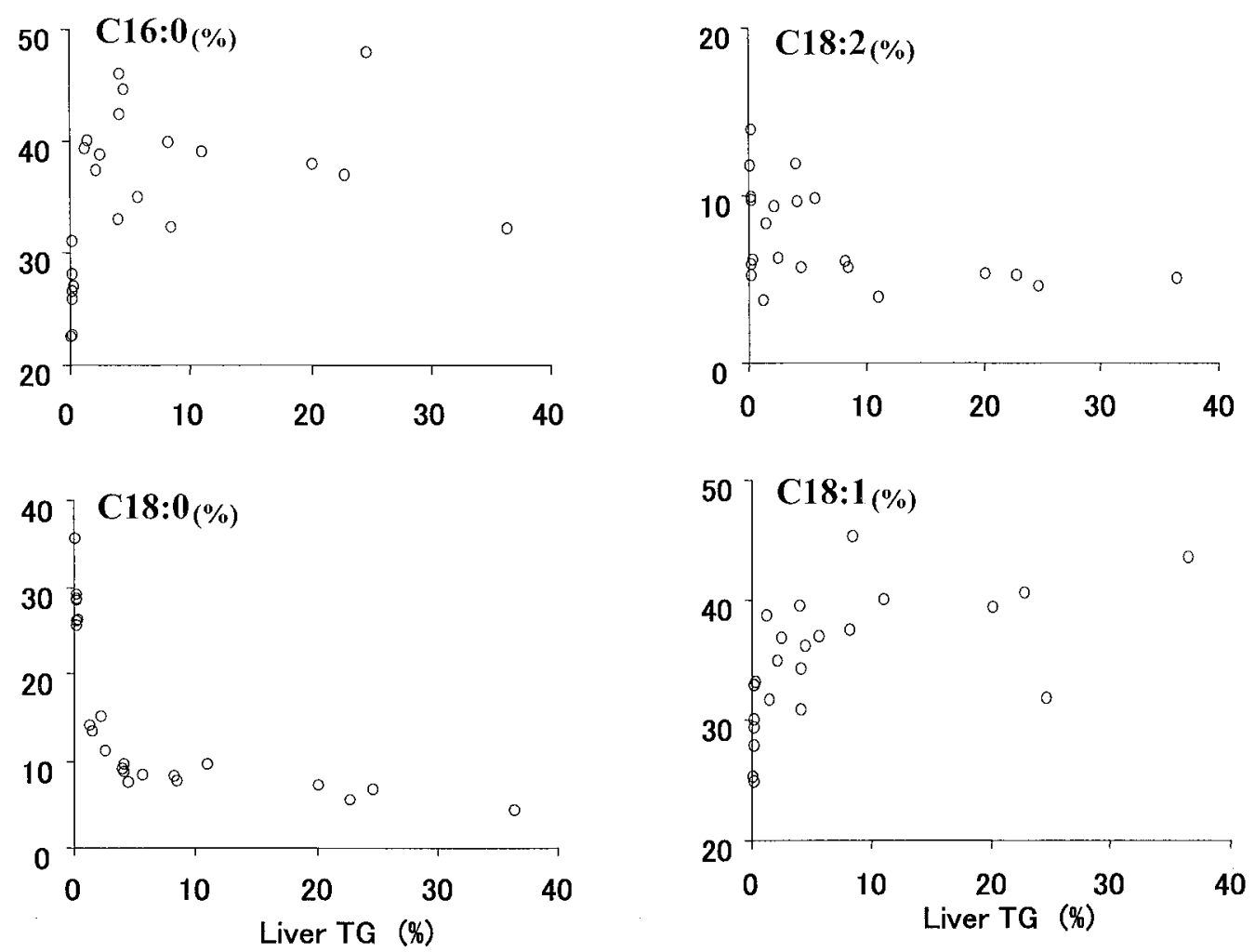

Fig. 1. Relationship between hepatic TG levels and the percentage of each fatty acid in the liver of all the cows. C16:0 Palmitic acid, C18:0 Stearic acid, C18:1 Oleic acid, C18:2 Linoleic acid. The cases having hepatic TG levels greater than $3 \%$ were categorized as fatty liver cows.

healthy cows. In myristic, palmitoleic, linoleic and linolenic acids, no significant differences were observed between the healthy and fatty liver cows $(P>0.05)$. Hepatic fatty acid profiles of healthy cows in the present study were comparable to those described in perirenal adipose tissue in Holstein steers [12].

Relationships between liver TG levels and the various fatty acid proportions are depicted in Fig. 1. The proportion of stearic acid decreased markedly with increased liver TG, but proportional variation of stearic acid was minimal in the fatty liver cows. Conversely, palmitic and oleic acid proportions rose with increased liver TG and fluctuated widely. Although no clear tendency was shown in linoleic acid proportions in relation to shifting levels of liver TG, some of the cows with low levels of liver TG had somewhat higher proportions of linoleic acid.

The most striking finding of this study was the proportionately sharp decline in stearic acid in fatty liver in conjunction with elevated levels of palmitic acid and oleic acid. One of the characteristics of ruminant liver lipid is a high stearic acid content and a low oleic acid content [10]. Proportions of the various fatty acids in the liver are influenced basically by the liver uptake of fatty acids from the circulating blood and by their metabolism, i.e., de novo synthesis, desaturation and chain elongation of fatty acids within the liver. Generally, synthesis $[1,4]$ as well as desaturation [1,
$2,10]$ of fatty acids is limited in the ruminant liver. Accumulation of palmitic acid in fatty liver in the present study seemed to coincide with weakened elongation of palmitic acid to stearic acid, however, the elongation activity is particularly weak in bovine liver [2]. In line with Rukkwamsuk et al. [7, 8], owing to the measurement of fatty acids from whole lipids of the liver, interpretation is confounded in the present study by the different fates of fatty acids in the presence in TG and those in phospholipids [3]. Under physiological circumstances, a strategic functional role of unsaturated fatty acids might be to regulate lipid fluidity because, by chemical nature, they preclude excessive accumulation of high melting temperature, or hard lipids. Under fatty liver conditions, increase of unsaturated fatty acid (e.g., oleic acid) could conceivably maintain suitable fluidity in the hepatocyte or its membrane.

Although the possibility cannot be ruled out that desaturation of stearic acid to oleic acid in fatty liver, extensive studies have established that the ruminant liver obtains most of its long chain fatty acids from circulating plasma NEFA $[1,3,4]$. Activated lipolysis and increased serum NEFA level after parturition have been reported to lead to a vast increase in hepatic accumulation of TG and a shift in the hepatic composition of the fatty acids, i.e., stearic acid percentage decreases and that of palmitic and oleic acids increases [7]. In dairy cows, serum NEFA level decreases 
with advancing postpartum days, causing a rebound in the proportions of liver fatty acids to a pattern similar to prepartum profiles [8]. Rukkwamsuk et al. [7] proposed that large amounts of stearic acid are used by the liver (e.g., oxidation) or secreted in milk, thereby proportionately lowering the hepatic stearic acid. In the present study, however, reduction of stearic acid in the liver cannot be explained by mammary secretion of the acid, because some of the fatty liver cows were not in lactation.

Plasma fatty acid profiles were not analyzed in the present study, but blood concentrations and uptake of the individual fatty acids are worth bearing in mind. Calving [6] or starvation [5] brings about a marked elevation of oleic acid among the various fatty acids in the blood plasma of ruminants. Moreover, specific differences in hepatic uptake of the individual fatty acids might exist. Early studies using noradrenaline infusion described the highest uptake of oleic acid by the liver, and the lowest uptake of stearic acid occurred in sheep [11]. Similarly, a linear relationship has been demonstrated between arterial concentration and the uptake of palmitic and oleic acids, while the uptake ratio of stearic acid was reported to be lower or variable regardless of arterial concentration of the acid [1]. The chemical properties or formula of individual fatty acid must be addressed to understand the movement of fatty acids through the circulating blood and the liver. Liver uptake of each fatty acid seems to be inversely related to the chain length in the case of saturated fatty acids and directly related to the number of double bonds of fatty acids (i.e., oleic $\geq$ palmitic $>$ stearic acid) [9]. In conclusion, differential uptake of the individual fatty acids by the liver could play an important role in governing the fatty acid profiles of the liver in dairy cows with fatty liver.

ACKNOWLEDGMENTS. The work was supported by a grant- in -aid for High Technological Research Center (Rakuno Gakuen University) from the Ministry of Education, Science, Sports and Culture of Japan. The authors wish to thank Prof. Kennedy, N. for her critical reading and improving the manuscript.

\section{REFERENCES}

1. Bell, A.W. 1981. pp. 363-410. In: Lipid Metabolism in Ruminant Animals (Christie, W.W. ed.), Pergamon Press, Oxford.

2. Drackley, J.K. 2000. pp. 97-119. In: Farm Animal Metabolism and Nutrition (D'Mello, J.P.F. ed.), CABI Publishing, Oxon.

3. Drackley, J.K., Overton, T.R. and Douglas, G.N. 2001. J. Dairy Sci. 84: E100-E112.

4. Emery, R.S., Liesman, J.S. and Herdt, T.H. 1992. J. Nutr. 122: 832-837.

5. Jackson, H.D. and Winkler, V .W. 1970. J. Nutr. 100: 201207.

6. Mulder, I. 1971. Biochem. J. 122: 12P-13P.

7. Rukkwamsuk, T., Geelen, M.J.H., Kruip, T.A.M. and Wensing, T. 2000. J. Dairy Sci. 83: 52-59.

8. Rukkwamsuk, T., Kruip, T.A.M., Meijer, G.A.L. and Wensing, T. 1999. J. Dairy Sci. 82: 280-287.

9. Soler-Argilaga, C., Infante, R. and Polonovski, J. 1973. Biochim. Biophy. Acta 326: 167-173.

10. St. John, L.C., Lunt, D.K. and Smith, S.B. 1991. J. Anim. Sci. 69: 1064-1073.

11. Thompson, G.E. and Darling, K.F. 1975. Res. Vet. Sci. 18: 325-327.

12. Tsuneishi, E., Nishimura, K. and Takimoto, Y. 1989. Anim. Sci. Tech. 60: 315-320. 